UDK 821.163.6-31.09.09Capuder A.:1(091)Bergson H.

\title{
HENRI BERGSON - LE MAÎTRE À PENSER DE L'GEUVR ROMANESQUE D'ANDREJ CAPUDER
}

\author{
Boštjan Marko Turk
}

\begin{abstract}
Résumé
La présente étude se donne pour objectif de porter un regard critique sur les éléments du bergsonisme présents dans l'ensemble de l'œuvre romanesque d'Andrej Capuder. En effet, ses romans sont ancrés dans l'expérience de la durée vécue ou la subjectivité, basée sur la négation du temps objectif que présente la coexistence de tous les termes bergsoniens impliqués dans l'œuvre. Le bergsonisme permet aux protagonistes de prendre, en s'appuyant sur l'élan vital, la conscience des aspects multiformes de leur propre personnalité face à l'Histoire.
\end{abstract}

Mot clefs : Herni Bergson, durée, élan vital, mémoire, roman, histoire, libération, amour, guerre, mort, système totalitaire

Le chemin d'Andrej Capuder, qui s'ouvre sur les premiers essais poétiques dans les années 1960, se poursuit avec de nombreuses traductions, dont celle de La Divine Comédie, ${ }^{1}$ couronné par le plus prestigieux prix de traduction slovène, ${ }^{2}$ témoigne à l'aube de son soixante-dixième anniversaire de la grande vitalité de l'auteur. La vigeur est le soubassement inhérent et inévitable de cette intensité subversive qui marque l'essentiel dans l'itinéraire de celui que le public slovène considère volontiers comme le plus grand connaisseur de la philosophie et de la littérature françaises au sein de l'Université de Ljubljana.

Le coup d'envoi initial de cette œuvre qui ne s'est jamais laissée assigner à demeure, on pourrait le chercher dans l'appréhension du bond épistémologique reposant sur l'opposition du temps, spatialisé par la gamme des outils que la commodité humaine peut imaginer, et la conscience de l'individu dans son sentiment de durer. Cette force étant tellement coextensive de la vie même qu'elle se présente comme un apriori de toutes les démarches de la pensée. À vrai dire, l'impulsion initiale n'a pas pu éviter l'ontologie en mouvement : les pages qui l'ont suivie cernent ses étapes et agissent comme un mot de passe guidant infailliblement à la destination.

\footnotetext{
${ }^{1}$ Dante Aligheri, Božanska komedija, Obzorja, Maribor, 1972.

${ }^{2}$ Le prix Sovre (Sovretova nagrada) décerné en 1973.
} 
Il a fallu le XXe siècle pour que Henri Bergson découvre l'écart entre la durée vécue et le temps réduit en espace. Ainsi l'œuvre romanesque d'Andrej Capuder - le poursuivant - ne tarde pas: elle en est imprégné littéralement dès ses premiers pages. En prenant en compte le genre modulable de sa trilogie dont le titre définitif ne sera jamais là, grâce au fait que «La réalité donnée, ce n'est pas être, c'est la durée pure, c'est le pur devenir $»,{ }^{3}$ on voit se dresser, sur cette ligne d'action proposée en vue épistémologique, avec les retombés bien au-delà de la philosophie, presque érigée en maxime la mise en regard métaphorique de la pensée bergsonienne, surprise in situ : «Les traces de la passion qui soutenait l'organisme vivant, faisant battre son cour, palpiter l'arbre artériel et agiter les muscles et la chair, ne disparaissent pas soudainement du visage de la dépouille mortelle, semant, en quelques instants, à tout vent l'horreur, la destruction et la mort; pourtant petit à petit les rides s'aplatissent irréversiblement : la chaleur disparait et pas à pas le bruit lointain du poumon vidé fait preuve définitive que nous assistons désormais à un corps privé des derniers signes de la vie - l'impression de la sorte remplissait les Italiens qui poursuivaient, avec précaution et non sans soucis l'exode massif des troupes Austro-hongroises. Pourtant, leur peur, cette fois-ci, était inutile. Le fauve a péri pour jamais». ${ }^{4}$

Les traits de la passion vitale qu'on décèle sur le visage d'un défunt, comme dernier signe de la vie disparaissant, est l'image qui illustre les passages focaux de L'Évolution créatrice. En fait, l'œuvre majeure d'Henri Bergson explique les postulats fondamentaux de sa philosophie, à commencer par les idées générales du processus évolutif, continuant par l'intelligence et l'instinct, terminant par la vie et la conscience et surtout la place de l'homme en face de soi et en face de l'espace qui l'entoure. ${ }^{5} \mathrm{Au}$ milieu nous retrouvons le passage parabolique : "Et notre plus ardent enthousiasme, quand il s'extériorise en action, se fige parfois si naturellement en froid calcul d'intérêt ou de vanité, l'un adopte si aisément la forme de l'autre, que nous pourrions les confondre ensemble, douter de notre propre sincérité, nier la bonté et l'amour, si nous ne savions que le mort garde enocore quelques temps les traits du vivant $\gg .{ }^{6}$

L'identité de l'image parabolique inaugurant la trame de Rapsodija 20 et le passage explicatif de L'Évolution créatrice est loin d'être une coïncidence. L'œuvre romanesque d'Andrej Capuder est en fait la confrontation de la durée vécue et le temps réduit en espace, celui-ci étant représenté par la rigueur mécanique, ce qui est la force que ses protagonistes ne peuvent pas peser dans la balance. Elle se manifeste en dernière instance comme la marque distinctive des êtres humains se traduisant par la conscience de périr et les contours palpables de l'anéantissement, la mort. Dans sa forme perpétuelle, elle englobe les héros des romans, poursuivant leur chemin à travers la diachronie la plus immédiate de l'espace, situé au niveau de l'individualité de l'autrui, c'est-à-dire, l'histoire. Le contact de l'individu et l'espace vital de son existence pourrait être désigné par le mot « endosmose ». Ainsi : «Or, entre cette succession sans extériorité et cette extériorité sans succession une espèce d'échange se produit, assez analogue à ce

\footnotetext{
${ }^{3}$ Cresson André, Bergson. Presses universitaires de France, Paris, 1961, p. 41.

${ }^{4}$ Andrej Capuder, Rapsodija 20, Slovenska matica, Ljubljana, 1982, p. 9.

${ }^{5}$ Henri Bergson, «L'Évolution créatrice ». CEuvres. Presses universitaires de France, Paris, 1970, pp. 578-650.

${ }^{6}$ Henri Bergson, Euvres, p. 603.
} 
que les physiciens appellent un phénomène de l'endosmose ». ${ }^{7}$ La phénoménologie de l'être humain, pris dans le cycle romanesque d'Andrej Capuder, repose sur la pulsation constante que constitue l'écart entre l'action extérieure englobant les circonstances dans lesquelles se trouve impliquée une destinée particulière dans un temps (successions des moments extérieurs) et le recul dans la simultanéité que présentent les moments infinis et innombrables de la conscience humaine. La lutte est désormais sans merci : « Notre entité profonde se situe à un niveau différent, au niveau de la durée qu'il nous faudra davantage extraire des entraves de l'espace $\gg .{ }^{8}$

En effet, l'extraction " des entraves de l'espace » est un socle sur lequel s'érigent les vastes fresques dans l'œuvre, les portraits des personnes, des relations et des conflits qui sont dès le début compris dans un titre qui indique nettement la limitation du temps par l'étendue indéfinie. En fait, le syntagme Rapsodija 20 que l'on rencontre dès le frontispice, loin d'être un cryptogramme ou un intitulé qui rendrait le contenu des livres plus compréhensible en le simplifiant, juxtapose deux éléments qui sont les moteurs puissant de la dialéctique bergsonienne. Le nombre « 20 » traduit les limites exactes de l'exitus et redditus dans lesquelles apparaissent les protagonistes de la première partie du cycle. En réalité l'image du fauve ayant péri, déjà évoqué, indique la fin de l'époque monarchique et le début du XXe siècle, à la fois. Il porte encore les « traces du vivant » puisque, dorénavant les souvenirs et les empreintes que la période a laissés sur les générations qu'elle a consummée, continueront à subsister sous une forme plus épurée, se présentant comme une suite divergente des simultanéités, c'est-à-dire, la durée. L'autre bout de ce temps prismatique sera à deux décennies du premier, à la fin du long cycle qui sépare l'armistice de 1918 et le 1 septembre 1939, un des moments culminant du siècle passé.

En ce qui concerne le substantif compris dans le syntagme, la rhapsodie, il faut d'abord rappeler qu'il désigne une suite de phrases musicales libres et une inspiration pouvant englober aussi bien des sujets populaires que des sujet plus épiques, modernes et sophistiqués. Cette pièce instrumentale illustre brillamment la sensibilité bergsonienne. En effet, la mélodie est l'image favorite, par laquelle le philosophe exprime son terme majeur. Andrej Capuder insiste sur ce point. «L La mélodie ou la phrase musicale est pour Henri Bergson une des images favorites de la durée, puisque tout dépend, chez elle, de l'état de notre vigilance: si nous voulons, c'est un organisme, si nous ne le voulons pas, ce n'est que la suite des notes clairsemées. En plus, la mélodie est un évocateur excellent, capable de rappeler intégralement les tréfonds de notre passé ».9

Dans le passage cité, on voit la prédilection de l'auteur pour l'appréhension du temps vécu par la conscience, phénomène par lequel se distingue infailliblement tout le dynamisme de la réalité humaine. Bergson revient continuellement sur le flux ininterrompu qui s'écoule entre les bornes du temps linéaire. Il peut être reconstruit par l'homme grâce à l'analogie avec une phrase musicale qui devient une mélodie. Voici un des extraits les plus significatifs : "La durée toute pure est la forme que prend la succession de nos états de conscience quand notre moi se laisse vivre, quand il s'abstient

\footnotetext{
${ }^{7}$ Henri Bergson, Euvres. p. 73.

${ }^{8}$ Andrej Capuder : «Henri Bergson ali osmišljena materija ». Esej o smehu, Slovenska matica Ljubljana, Ljubljana, 1977, p. 197.

${ }_{9}^{9}$ Andrej Capuder : « Henri Begson ali osmišljena materija », p. 200.
} 
d'établir une séparation entre l'état présent et les états antérieurs. Il n'a pas besoin pour cela, de s'absorber tout entier dans la sensation ou l'idée qui passe, car alors, au contraire, il cesserait de durer. Il n'a pas besoin, non plus d'oublier les états intérieurs : il suffit qu'en se rappelant ces états il ne les juxtapose pas à l'état actuel comme un point à un autre point, mais les organise avec lui, comme il arrive quand nous nous rappelons, fondues pour ainsi dire ensemble, les notes d'une mélodie $» .^{10}$

On s'aperçoit que, dans le roman, l'idée du titre intervient, dans sa fraîcheur toujours renouvelée, lorsque l'auteur propose la synthèse des événements passés. Ces moments de l'œuvre fonctionnent à la fois comme une prise de repères sur lesquels s'organise l'évolution du texte révolu, et comme une sorte de phare jetant la lumière sur ce que le lecteur va encore découvrir. Rapsodija 20 est une œuvre organisée de façon ingénieuse, pourvue de structures qui ne pourraient pas exister sans la pensée bergsonienne. On les retrouve aux pivots focaux, à la fin ou au début de l'un ou l'autre chapitre qui, eux aussi, traduisent formellement la même idée de reprise et de contemplation. Le roman s'ouvre notamment sur une fresque savamment reprise dans l'image du dénombrement homérique déjà évoquée précédemment, pour avancer vers la scène, à la fin du chapitre VI, où le vieux France Neubauer, l'ancien et le futur maire de la capitale slovène, dresse le bilan de la période, de sa vie et de la nation entière. L'instant est finalement venu pour le petit peuple slovène, coincé entre trois grandes nations européennes, de fêter son indépendance relative ; les cris dans les rues suscitent chez l'écrivain des impressions qu'il résume succinctement : "Ils buvaient et chantaient, se réjouissaient et pleuraient. Entre le vin répandu sur les tables et les tessons des bouteilles prenait la fin l'époque de l'asservissement millénaire ». ${ }^{11}$

L'endosmose est l'espace du temps objectif mesuré pour les besoins du roman par les débris de l'empire et des bouteilles, ce qui constitue une image, un rapprochement, et non l'expression exacte. De l'autre côté, il y a le temps subjectif du héros France Neubauer qui se traduit par l'idée de la phrase musicale, soigneusement introduite par la rencontre du vieil homme et l'instrument préféré de sa femme, le piano. Le maire de Ljubljana a perdu son épouse durant l'été 1914, pendant la saison qui marque le commencement des deux décennies et du XXe siècle, comme nous l'avons déjà dit précédemment. De ce décor homogène, il descend en soi pour retrouver l'objet de son adoration ou, mieux, pour lui exprimer ses sentiments : "Son intérieur a étéfracassé suite à la quête ... de la parole qui pourrait en même temps excuser, expliquer, accuser, se plaindre et apporter du réconfort mais surtot boucler le cercle: mémoire et actualité, trépas et occurrence, faute et absolution, enfin, lui et la défunte. Il avait le pressentiment de ce qu'il était en train de dire, mais soudainement la honte l'envahit. En face de tant de tombeaux, de tenaillement et de saleté, au-dessus de la tranchée, à travers les barreaux des cellules, de cette maison dévastée, une attrition descendait sur lui. Il avait honte à proférer ce qu'il voudrait, de tout son cœur et de toute son âme. Sa main est retombée sur le clavier. Le ton nouveau qui naissait renforcé était comme une écluse qui relâchait l'amas des quantités d'eau jusqu'alors inimaginables. Les souvenirs obnubilés par les années réapparaissaient, lui montrant l'image des cheveux noirs sur une épaule blanche, de la poitrine et de sa taille élégante. Tout d'un coup, il a décidé

\footnotetext{
${ }^{10}$ Herni Bergson, Euvres, p. 67.

${ }^{11}$ Andrej Capuder, Rapsodija 20, p. 32.
} 
de l'appeler. Avec un effort visible il a desserré la mâchoire pour articuler ce qu'il ne lui avait jamais dit de son vivant : "Amour $» .^{12}$

L'endosmose de la philosophie de la durée, appuyée sur le fécond appareil des notions, parmi lesquelles une place prestigieuse est vouée à la «mélodie », n'est pas toujours explicitement présente dans le roman. Elle peut, au contraire, être aisément déduite du contexte ; pourtant aux points incontournables, là où la trame du texte se cristallise de façon à laisser entrevoir le futur arrangement des événements aussi bien qu'à récapituler ce qui s'est passé jusqu'à ce moment-là, l'apperception immédiate des termes bergsoniens ne tarde pas à apparaître dans sa brillante splendeur. Notamment, il existe un passage au milieu du livre, à la fin du chapitre intitulé « L'homme du Conseil de Trente $»,{ }^{13}$ où les idées de l'écrivain prennent corps sans équivoque, enrichissant ce qu'on a découvert jusque là en introduisant le concept suprême de la philosophie bergsonienne, c'est-à-dire l'image. "Corpus est mens momentanea sed carens recordatione $»^{14}$, note Albert Thibaudet, l'un des plus profonds connaisseurs de cette philosophie. Cela signifie qu'il manque au corps, celui-ci étant équivoque d'un objet, quelque chose comme une ancre pour s'engager dans la simultanéité d'un temps non spatialisé. Cet outil, c'est l'image dotée d'un rôle ontologique : "Les images, c'est l'être même, tel que l'intuition de la durée pure nous le révèle en nous et tel que la sympathie divinatrice nous le révèle partout $\gg .{ }^{15}$ L'image désigne en extension ce qui est au-delà de la limite d'être dénoté ou conceptualisé : "L'image est ainsi un symbole non conceptuel le symbole de ce qui ne peut pas être symbolisé : tout symbolisme a sa validité tant qu'il expose une vue sur les choses : appliqué au moi, il devient image et l'image sera rendue à son vrai sens si on la delivre du symbolisme pour lui rendre sa fonction propre : être la manifestation de l'activité symbolique fondamentale du moi qui dure $»{ }^{16}$ L'image, appliquée au moi est l'indice de l'égalité vérifiée pour toutes les valeurs que sous-entendent les signifiants qui la constituent. Les mots-clefs de Rapsodija 20 peuvent sans encombre se ramener à la spatialisation du temps linéaire qui engloutit les personnages, c'est-à-dire à l'histoire. Il est vrai que les deux décennies $(1918$ - 1939) sont l'espace où règne la paix. Pourtant, celle-ci n'est que provisoire : le roman commence par la fin d'un carnage et est comme un pont jeté jusqu'à un autre massacre sanglant. La parabole homérique de l'armée dissoute d'un côté et le suicide de France Neubar à la fin de l'autre se tiennent la main : à l'intérieur de la paume, il y a de la souffrance, de la violence et de la mort charnelle. "Toute perception est conscience d'images, dont pour chacun, l'image privilégiée de son corps $\gg .^{17}$ De ce point de vue, l'image appliquée au moi, ne surprend pas. C'est la citadelle ${ }^{18}$ qu'on assiège. Dans les batailles qui se déroulent à l'infini, si on prend comme mesure temporelle la durée de l'existence humaine, pendant des siècles. On n'en entrevoit pas la fin, puisqu'elle n'est pas concevable et là commence à s'esquisser

\footnotetext{
${ }^{12}$ Andrej Capuder, Rapsodija 20, p. 37. .

${ }^{13}$ Andrej Capuder, Rapsodija 20, p. 131.

${ }^{14}$ Albert Thibaudet, Le Bergsonisme, Paris, Nouvelle Revue Française, 1924, p. 88

${ }^{15}$ André Cresson, Bergson, Presses universitaires de France, Paris, 1961, p. 35. .

${ }^{16}$ Pierre Trotignon, L'Idée de vie chez Bergson et la critique de la métaphysique, Presses universitaires de France, Paris, 1986, p. 610.

${ }^{17}$ Bernard Gilson, L'Individualité dans la philosophie de Bergson, Librairie philosophique J. Vrin, Paris, 1985 , p. 21.

${ }^{18}$ Le terme dont se sert l'auteur est « trdnjava ».
} 
l'analogie avec l'identité foncière du héros principal. C'est une image qui est à la fois le souvenir vivace des événements vécu et la promesse de tout ce qui viendra encore, bref, l'image de la durée : "C'était lui, l'attaquant esseulé d'une citadelle imprenable qui lui avait été confié comme une sorte de destin : l'assiéger et l'enlever d'assaut : elle était à la fois son but et sa mémoire, à vrai dire, sa propre personnalité, lui seul. Il a mis longtemps à parvenir à cette connaissance. Les siècles devaient passer jusqu'à ce que la grêle des projectiles eût rempli sa chair afin de s'y reposer finalement. Il en fallait autant pour que ses murs fortifiés et garnis de ferrures fondent sous l'effet de son désir brûlant, visant le but. Il en fallait autant afin que la lueur rouge, associée au son perçant des trompettes et d'autres instruments de musique militaires se fût désobjectivisée $e^{19}$ dans sa présence triomphante. Son cœur battait, lorsque sa main s'étendait jusqu' à l'avenir infini, pour cueillir le laurier de victoire. Qui ou quoi verra-t-il sur la passerelle de commandement, la haut, d'où on menait la défense et l'assaut, la démolition et l'assemblage, la victoire et la défaite de celle qui était sa vie même $»{ }^{20} \mathrm{Le}$ passage décèle l'épistémologie entitative du narrateur; il est de prime importance, ${ }^{21}$ puisque il permet d'entrevoir la nature secrète de l'organisateur de l'œuvre : à travers les siecles son individualité, basée sur l'expérience du corps, c'est-à-dire les sensations, se dilate, échappant aux lois du temps homogène. Son regard réflexif se porte sur les mouvements immédiats de la durée et emprunte le chemin reliant la perception passive à la représentation active, comme une nébulosité qui se condense de plus en plus pour passer à l'état actif afin de remporter la victoire. Il s'agit du « mouvement par lequel le passé s'épanouit en image présente, émergeant des ténèbres au grand jour $\gg{ }^{22} \mathrm{En}$ fait, c'est «l'attention à la vie $»^{23}$ qui est la puissance motrice de l'image où le narrateur et le héros principal sont réunis dans une même entitée ${ }^{24}$ témoignent bien que "le symbolisme naît de la spatialisation du vécu de la conscience $»,{ }^{25}$ dans le temps sériel, car l'espace homogène est le symbole des symboles surtout quand on l'applique à des couches de plus en plus intimes d'un moi.

La ligne d'inspiration qu'a exercée Henri Bergson sur la créativité romanesque d'Andrej Capuder continue à être présente dans les autres romans. Si nous abordons l'œuvre suivante, Iskanje drugega (La Quête de l'autre), nous voyons que le paradigme des images qui sont la réfraction du moi profond à travers ses épiphénomenes superficiels, persiste en prenant encore plus de consistence. Notamment, l'ossature du texte est basée sur l'attente et finalement sur l'avènement de tertia aetas, le temps à la fois

\footnotetext{
${ }^{19}$ Le terme employé dans le texte est « razpredmetenje ».

${ }^{20}$ Andrej Capuder, Rapsodija 20, p. 143.

${ }^{21} \mathrm{C}$ 'est un jugement de valeur qui porte sur l'ensemble du livre.

${ }^{22}$ Henri Bergson, CEuvres, p. 179.

${ }^{23}$ Henri Bergson, Euvres, p. 166, les corrélatifs slovènes de ce paradigme intentionnel sont : « cilj », « vroče poželenje », « zmagovita vseprisotnost ».

${ }^{24}$ Concernant le mécanisme de l'unification auquel se réfère principalement le texte, voir : « Mais nous arrivons naturellement à cette représentation symbolique par ce seul fait que, dans une série de termes identiques, chaque terme prend pour notre conscience un double aspect : l'un toujours identique à lui-même, puisque nous songeons à l'identité de l'objet extérieur, l'autre spécifique, parce que l'addition de ce terme provoque une nouvelle organisation de l'ensemble. De la possibilité de déployer dans l'espace, sous forme de multiplicité numérique, ce que nous avons appelé une multiplicité qualitative, et de considérer l'une comme l'équivalent de l'autre », Henri Bergson, Euvres, p. 82.

${ }^{25}$ Pierre Trotignon. L'Idée de vie chez Bergson. Paris: Presses universitaires de France. 1968. P. 607.
} 
eschatologique et entitatif, représenté par le symbole de la porte qui s'ouvre au héros principal à la fin du roman. La fin du texte, c'est la réalisation de son commencement dont l'espace ne pourrait conserver aucune trace, la volonté de l'artiste étant de remplacer l'idée de succession par la durée sérielle. Cette démarche dénote l'identité du livre entier, ne laissant pas de doute sur la source de l'inspiration. Henri Bergson l'a énoncé dans l'image anthologique de ses réflexions constituée par l'oscillation. «Je les apercevrai (les oscillations, remarque, B.M.T.) l'une dans l'autre, se pénétrant et $s$ 'organisant entre elles comme les notes d'une mélodie, de manière à former ce que nous appellerons une multiplicité indistincte ou qualificative, sans aucune ressemblance avec le nombre : j'obtiendrai ainsi l'image de la durée pure, mais je me serai entièrement dégagé de l'idée d'un milieu homogène ou d'une quantité mesurable ». ${ }^{26}$

Le temps n'est pas la mesure d'une répétition dans l'espace puisque, à ce moment-là, il n'y avait ni la conscience de ce qui s'est passé autrefois, ni celle de l'avenir inévitable, ni celle du présent dans lequel plonge le roman. ${ }^{27}$ Ainsi, à l'image de la citadelle évoquée plus haut se joint l'idée du temps qui est essentielement bâti sur la perception sans cesse actualisée de l'existence. Dans Iskanje drugega, la démarche bergsonienne permet de revenir régulièrement en arrière, au point initial où tout a commencé. "Il avait peur de ne pas forniquer lorsqu'il se penchait, dans une sorte de volupté aveugle, sur sa mémoire, voulant en extraire et par conséquent aussi sauvegarder dans la durée tous les sentiments relatifs au passage de l'état de péché à celui de grâce. De l'autre côté, un instinct irrésistible lui suggérait qu'il fallait revenir au commencement même, à la croisée initiale des chemins où il s'était, pour la premiere fois, posé la question quod iter sectabor vitae? C'était le point de départ où l'âme a premièrement flambé dans la liberté de pouvoir choisir, ce qui a constitué son acte de naissance $» .{ }^{28}$ En fait, le passage cité, extrait du chapitre central intitulé «Quod iter sectabor vitae $»,{ }^{29}$ est mis dans un rapport compréhensif avec le professeur du Collège de France par Andrej Capuder lui-même. La délibération sur laquelle insiste tant Marko Neubauer, le personnage principal du récit (lui, par ailleurs, joue un rôle prépondérant dans toute la série des œuvres qui se succèdent dans l'épiphénoménologie de la durée) a été savamment explorée dans le cours sur le classicisme et sur l'évolution du sentiment romantique en France que l'auteur a publiée à la Faculté des lettres de Ljubljana afin d'expliquer au public universitaire slovène les principes de la philosophie des données immédiates de la conscience tels que l'exploitait la littérature du temps de Bergson et même antérieure. ${ }^{30}$ Le problème du verbe latin dont la forme est passive et le sens actif (sector) permet d'approcher au mieux le dilemme de l'autonomie dans le choix, compte tenu de l'hésitation entre deux actions possibles où le héros persévère dans l'illusion que le retour à la croisée initiale du chemin serait toujours possible. C'est l'erreur de perception que provoque l'apparence trompeuse de l'acte accompli qui se substitue, dans une sorte de mensonge caché, à l'action s'accomplissant, faisant vider la question

\footnotetext{
${ }^{26}$ Herni Bergson. Euvres. Paris: Presses universitaires de France. 1970. P. 70.

${ }^{27}$ "Iskanje drugega commence et fini dans le présent », Andrej Capuder. Rapsodija 20, couverture.

${ }^{28}$ Andrej Capuder. Iskanje drugega. Ljubljana: Slovenska matica. 1991. P. 233.

${ }^{29}$ Ibiden.

${ }^{30}$ Andrej Capuder. Du Classique au romantique. Ljubljana: Filozofska fakulteta Univerze v Ljubljani. 2000.
} 
du sens et permettant au problème de la liberté de sortir intact de la discussion. Ainsi : «La réponse de Bergson est paradoxale. Nous sommes libres quand nos choix dérivent de notre moi profond, d'une décision personnelle qui engage tout notre être. Savoir se placer dans la durée personnelle - et universelle - qu'est ce que c'est? C'est savoir demeurer dans la disposition de choisir, dans une ouverture de l'être où nous sommes plutôt choisis ». ${ }^{31}$

On dit que Marko Neubauer a choisi la chartreuse : en fait, il n'a délibéré qu'en apparence, puisque il a été déjà choisi par un apriori auquel son moi profond avait acquiescé sans réflechir, c'est-à-dire la durée. Il a fui la forme la plus cruelle de l'espace bergsonien, l'histoire, mieux, sa configuration particulière, le communisme et ses crimes. De même, Jernej, le protagoniste du roman Mali cvet (La Petite Fleur), se retire dans la musique, la forme favorite de la conscience extratemporelle du vécu, cette constance pérenne, privée de l'espace. Le moi profond choisi en étant le mieux choisi : cela, nous le devons aux données immédiates de la conscience. Andrej Capuder a effectué le même choix dans les conditions historiques d'un systeme totalitaire auquel il a été livré sans merci.

Cependant, le sacrifice n'a pas été vain. La rencontre inoubliable avec le bergsonisme n'a pas seulement élevé l'auteur, mais également la littérature slovène. En effet, celle-ci peut désormais se reconnaître dans l'inscription gravée sur l'un des piliers du Panthéon à Paris: «À Henri Bergson, le philosophe dont l'œuvre et la vie ont honoré la France et la pensée humaine ».

Université de Ljubljana, Slovénie

\section{BIBLIOGRAPHIE}

Barlow, Michel. Henri Bergson. Paris : Editions universitaires, 1966.

Bergson, Henri. Oeuvres. Paris : Presses universitaires de France, 1970.

Capuder, Andrej. "Henri Bergson ali osmišljena materija". Henri Bergson. Esej o smehu. Edité par Janez Gradišnik. Ljubljana: Slovenska matica, 1977. 193-262.

. Du Classique au romantique. Ljubljana, Filozofska fakulteta Univerze v Ljubljani, 2000.

. Henri Bergson, intuicija in misel. Ljubljana: Mohorjeva založba, 2008.

. Iskanje drugega. Ljubljana: Slovenska matica, 1991.

. Mali cvet. Ljubljana: Slovenska matica, 1977.

. Rapsodija 20. Ljubljana: Slovenska matica, 1982.

. Reka pozabe. Ljubljana: Slovenska matica, 2007.

Cresson, André. Bergson. Paris : Presses universitaires de France, 1961.

Deleuze, Gilles. Bergsonisme. Paris : Presses universitaires de France. 1966.

Gilson, Bernard. L'Individualité dans la philosophie de Bergson. Paris : Librairie philosophique J. Vrin, 1985.

Trotignon, Pierre. L'Idée de vie chez Bergson et la critique de la métaphysique. Paris: Presses universitaires de France, 1968.

Turk, Boštjan Marko. Bergsonizem in njegov položaj v duhovni zgodovini Slovencev. Ljubljana: Scripta, 2000.

${ }^{31}$ Andrej Capuder. Du Classique au romantique. P. 36. 